\title{
Evidence for Majorana phases in the magnetoconductance of topological junctions based on two-dimensional electron gases
}

\author{
Llorenç Serra $\circledast^{1,2}$ and Kaveh Delfanazari $\oplus^{3,4}$ \\ ${ }^{1}$ Institute of Interdisciplinary Physics and Complex Systems IFISC (CSIC-UIB), E-07122 Palma, Spain \\ ${ }^{2}$ Department of Physics, University of the Balearic Islands, E-07122 Palma, Spain \\ ${ }^{3}$ Electrical Engineering Division, Engineering Department, University of Cambridge, Cambridge CB3 OFA, England \\ ${ }^{4}$ Department of Physics, Cavendish Laboratory, University of Cambridge, Cambridge CB3 OHE, England
}

(Received 5 December 2019; revised manuscript received 20 February 2020; accepted 24 February 2020; published 9 March 2020)

\begin{abstract}
We calculate the linear conductance of a two-dimensional electron-gas-based junction between a normal semiconductor section and a hybrid semiconductor-superconductor section, under perpendicular magnetic field. We consider two important terms often neglected in the literature, the magneto-orbital and transverse Rashba spin-orbit. The strong orbital effect due to the magnetic field yields topological phase transitions to nontrivial phases hosting Majorana modes in the hybrid section. The presence of a potential barrier at the junction interface reveals the Majorana phases as quantized plateaus of high conductance, for low values of the chemical potential. In wide junctions (or large chemical potentials) the phase transitions occur at low magnetic fields but the magneto-conductance becomes anomalous and lacks clearly quantized plateaus.
\end{abstract}

DOI: 10.1103/PhysRevB.101.115409

\section{INTRODUCTION}

Majorana modes in nanostructures have been attracting strong interest since the experiments in semiconductor nanowires gave initial evidence on their real existence [1-5], in agreement with earlier theoretical studies (see Refs. [6-11] for reviews). Additional experimental evidence has been also obtained more recently [12-19]. In semiconductor nanowires, Majorana states at the end points of the wire can be engineered by combining three essential ingredients: Spin-orbit interaction, magnetic field and superconductivity. While the spin-orbit coupling is intrinsic to the semiconductor, the other two ingredients are not; superconductivity can be induced by proximity with a nearby bulk superconductor and the magnetic field has to be tuned externally. Although quantum wires are, ultimately, idealizations of one-dimensional (1D) systems, it soon became of interest to theorists the relevance of multibands in quasi-1D (q1D) nanowires with transverse degrees of freedom; either two-dimensional (2D)-q1D strips with a lateral width [20-25], or 3D-q1D nanowires with a given shape of the transverse cross section [26-29].

The 2D-q1D geometry is of special interest, as it relates to semiconductor 2D electron gases (2DEG's) of widespread application in semiconductor nanodevices. The induced supercondutivity in 2DEG's and planar Josephson junctions was demonstrated in Refs. [30-36]. In this planar geometry, a perpendicular magnetic field has a paramount influence on the motion of quasiparticles in the plane; the scenario of the well-studied quantum Hall effect. In our context of hybrid semiconductor-superconductor systems, the relevance of the magneto-orbital effect for the characterization of topological phases has been studied in different geometries; cylinders
[37,38], faceted wires $[27,28]$, and $2 \mathrm{D}$ strips or ribbons [39-41]. In 2D strips it is generally assumed that a perpendicular field is detrimental for the Majorana modes and a parallel field is more often considered where the magnetic effect is restricted to a Zeeman coupling with the quasiparticle spin. In Ref. [39] it was shown that when tilting the field from the horizontal orientation towards the vertical orientation there are critical angles beyond which observing the Majorana mode is no longer possible for weak SO coupling. Remarkably, however, it was predicted that with stronger couplings (relative to the transverse confinement energy) there are parameter ranges where end Majorana modes may be present even in fully perpendicular fields. For a theoretical study, a disadvantage of the perpendicular field in 2D-q1D geometry is that the determination of the topological transitions is not known analytically but only numerically; approximate analytical limits require a few-band truncation [40]. On the other hand, as mentioned above, the perpendicular field geometry is more convenient experimentally since, in this case, the field has a maximal influence and thus lower fields are more effective for parameter tuning.

In this work we analyze the topological phase diagrams of 2D-q1D strips in the presence of vertical field. We show that the magneto-orbital effect leads to nontrivial Majorana phases for relatively low values of the field, depending on the chemical potential and intensity of the SO coupling. We then consider transport in 2D-q1D junctions between a normal semiconductor and a hybrid semiconductor-superconductor, with the purpose of identifying signatures of the nontrivial Majorana phases of the hybrid strip in the linear conductance of the junction. We find that for low values of the chemical potential the junction conductance clearly reveals the topological phases as quantized conductance plateaus, robust against the 
presence of an interface potential barrier. On the contrary, the linear conductance of the trivial phases is severely quenched by an interface barrier. This clear difference in conductance between trivial and topological phases is restricted to relatively low chemical potentials, while it degrades for higher values. We then consider wider junctions, where the SO becomes stronger relative to the transverse confinement energy, showing that the topological regions shrink toward zero field; i.e., the sequence of multiple transitions trivial-topologicaltrivial, and so on, takes place at lower fields than in narrow junctions. On the whole, our results clarify the theoretical scenario for topological transitions of hybrid 2DEG strips in vertical magnetic fields, specifically showing how the junction magnetoconductance reveals such topological phases.

\section{MODEL}

We use the model of a hybrid semiconductingsuperconducting 2D-q1D wire (as reviewed, e.g., in Ref. [11]). Quasiparticle motion in the $x y$ plane is described with continuum coordinates $(x, y)$, with $y$ restricted to $-L_{y} / 2<y<L_{y} / 2$, i.e., a strip of width $L_{y}$. Spin and electron-hole (isospin) degrees of freedom are treated as discrete quantum variables with $\sigma_{x y z}$ and $\tau_{x y z}$ Pauli matrices, respectively. The Hamiltonian reads

$$
\begin{aligned}
\mathcal{H}= & \left(\frac{p_{x}^{2}+p_{y}^{2}}{2 m}-\mu\right) \tau_{z}+\frac{\alpha}{\hbar}\left(p_{x} \sigma_{y}-p_{y} \sigma_{x}\right) \tau_{z} \\
& +\Delta_{B} \sigma_{z}+\Delta_{0} \tau_{x} \\
& +\frac{\hbar^{2}}{2 m l_{z}^{4}} y^{2} \tau_{z}-\frac{\hbar}{m l_{z}^{2}} y p_{x}-\frac{\alpha}{l_{z}^{2}} y \sigma_{y},
\end{aligned}
$$

where $\alpha, \Delta_{B}$, and $\Delta_{0}$ are the SO, Zeeman and pairing parameters, respectively. The Zeeman energy $\Delta_{B}$ is related to the field $B$ by $\Delta_{B}=g^{*} \mu_{B} B / 2$, where $g^{*}$ is the gyromagnetic factor. The last three terms of Eq. (1), depending on the magnetic length $l_{z}^{-2}=e B / \hbar c$, are the orbital field terms. The chemical potential is represented by parameter $\mu$.

We obtain below solutions of Schrödinger's stationary equation for a given energy $E$,

$$
(\mathcal{H}-E) \Psi\left(x y \eta_{\sigma} \eta_{\tau}\right)=0,
$$

where $\eta_{\sigma, \tau}=1,2$ are the discrete spin and isospin variables, respectively. We use the complex band structure approach, where the $x$ dependence of the wave function is expanded in a set of wave numbers $k$, including real (propagating) and complex (evanescent) modes. The $y$ dependence is described in a $1 \mathrm{D}$ grid of uniformly distributed points and a general wave function is represented as

$$
\Psi\left(x y \eta_{\sigma} \eta_{\tau}\right)=\sum_{k} C_{k} e^{i k x} \Phi_{k}\left(y, \eta_{\sigma} \eta_{\tau}\right),
$$

where the $\Phi_{k}$ 's are determined from the solution of the 1D $k$-dependent eigenvalue problem (see below) and the $C_{k}$ 's are the set of complex amplitudes representing a given state [11].

The $\Phi_{k}$ 's in Eq. (3) are the transverse states of an infinite homogenous strip. They characterize the strip band structure $\varepsilon(k)$, for real values of $k$, from the eigenvalue equation

$$
h_{k} \Phi_{k}\left(y \eta_{\sigma} \eta_{\tau}\right)=\varepsilon_{k} \Phi_{k}\left(y \eta_{\sigma} \eta_{\tau}\right),
$$

where the 1D Hamiltonian $h_{k}$ is obtained by the replacement $p_{x} \rightarrow \hbar k$ in the general Hamiltonian $\mathcal{H}$ of Eq. (1). The strip topological transitions are characterized by the $k=0$ gap closings [42,43], i.e., by the condition $\varepsilon_{0}=0$. Specifically, we will analyze below the $B-\mu$ phase diagram of the topological strip determining the $\varepsilon_{0}=0$ curves in the diagram. Besides, it is also of interest to determine the regions where the global gap, i.e., the eigenvalue $\varepsilon_{k}$ for some $k$ not necessarily zero, vanishes [44]. This global gap vanishing condition $\varepsilon_{k}=0$ defines sizable portions of the $B-\mu$ plane, the gapless regions, in addition to the mentioned $\varepsilon_{0}=0$ curves of zero measure in the plane. In the gapless regions there are propagating states at zero energy and, therefore, any localized zero mode like the Majorana mode will decay into those extended states.

In practice, we determined the eigenvalues and eigenstates of Eq. (4) numerically, discretizing the $y$ coordinate in a uniform grid and using sparse matrix diagonalization techniques [45]. This method is very efficient computationally and allows well-converged results with the use of large-enough grids ( $\gtrsim 100$ points). In the junction case the Hamiltonian parameters are no longer constant since we assume a vanishing pairing $\Delta_{0}=0$ for $x<0$; corresponding to the normal semiconductor. The scattering problem is then solved as in Ref. [46], determining the transmission and reflection probabilities corresponding to a given incident mode. Adding the contributions from all possible incident modes we determine the linear conductance $G$ as [47]

$$
G=\frac{e^{2}}{h}\left(N-R+R_{A}\right),
$$

where $N$ is the number of incident electron modes, $R$ is the normal reflection probability, and $R_{A}$ is the Andreev reflection probability. Equation (5) contains the well-known result that while normal reflections decrease the conductance, Andreev reflection processes, whereby incident electron quasiparticles are reflected as holes, enhance the conductance.

\section{RESULTS}

\section{A. Phase diagrams}

Figure 1 shows the $B-\mu$ phase diagrams of a hybrid strip of $L_{y}=150 \mathrm{~nm}$ for different values of the SO coupling $\alpha$. Since the value of $\alpha$ is sample dependent and it can be actually tuned with electric fields we chose some representative values within the typical range of InAs 2DEG's with the purpose of investigating the tendency for increasing or decreasing $\alpha$ [48]. The topological regions with one Majorana mode are indicated by the cyan lines and a letter M in Fig. 1. Considering, for instance, an evolution of parameters with constant $\mu$ and increasing $B$ from the trivial $B=0$ phase, there is a phase transition to a one-Majorana phase whenever a cyan line is first crossed. That is, the cyan lines are enclosing one-Majorana phases in a background of the trivial phase. A similar topology of the $B-\mu$ plane was already obtained in Ref. [49] but neglecting the terms in $\alpha p_{y}$ and all the orbital $l_{z}$ terms. We find that the shape of the transition borders is strongly affected by these terms that actually dominate for large enough fields. Remarkably, we also find a Majorana island surrounded by trivial phase in Fig. 1(b). 



FIG. 1. Phase diagrams of a hybrid strip with $\Delta_{0}=0.3 \mathrm{meV}$, $L_{y}=150 \mathrm{~nm}$ for different values of the SO coupling: $\alpha=$ (a) $60 \mathrm{meV} \mathrm{nm}$, (b) $45 \mathrm{meV} \mathrm{nm}$, and (c) $30 \mathrm{meV} \mathrm{nm}$. The cyan lines encircle the topological regions with one Majorana mode in the $\mu-B$ plane, obtained as gap closings for $k=0$ and signalled with a letter $\mathrm{M}$. The black line shows the boundary for activation of a propagating mode, separating the hatched region of any- $k$ gap closing in the upper right corner. The background color indicates the number of electron/hole propagating modes in the corresponding normal strip having $\Delta_{0}=0$ and the same rest of parameters $\left(N_{e h} \equiv\right.$ $2 N$ ). Additional parameters: $m=0.033 m_{e}, g^{*}=15$.

There is a clear correspondence in Fig. 1 between oneMajorana regions and regions with an odd number $N$ of electron propagating modes in the normal strip (the strip with $\Delta_{0}=0$ and the same rest of parameters). These last are shown by the colorscale background in Fig. 1, only minor deviations at very small fields can be observed between both types of regions. This relation is not obvious a priori and it was already pointed out in Ref. [49] as a correspondence between the topological number $|Q|$ of the hybrid system and the number of propagating modes $N$ of the normal one. We find that such correspondence is thus preserved by the magneto-orbital terms. The value of $N$ is relevant in the NS junction system (see below) since it determines the number of incident modes from the normal side. We also notice that all the $\mathrm{M}$ regions in Fig. 1 correspond to a topological number $|Q|=1$ Majorana mode.

The boundary for the activation of propagating modes in the hybrid system is indicated by the black line in Fig. 1; the gapless phase corresponding to the hatched upper right corner of each panel. In this gapless phase the Majorana mode looses its protection as it decays into propagating modes with the same zero energy. As mentioned above, the gapless phase is a sizable region where propagating modes exist for a specific finite (nonvanishing) $k$ and it should be distinguished from the cyan lines in Fig. 1 representing gap closing only for $k=0$.

\section{B. Magnetoconductance plateaus}

We now turn to calculating the magneto-conductance $G(B)$ when a normal lead is attached to a hybrid semi-infinite strip, forming a 2D-q1D junction [see sketch in Fig. 2(d)]. We will focus on the the small bias (linear) regime obtaining the conductance from Eq. (5) and including a potential barrier $V_{0}$ of length $L_{x}$ near the junction edge to tune the effective coupling between the $N$ and $h S$ parts. We choose a fixed value of $\mu$ and then sweep $B$ to probe the different system phases displayed in Fig. 1. The results of Fig. 2 show some representative cases; Fig. 2(a) corresponds to $\mu=$ $1.5 \mathrm{meV}$ in Fig. 1(a), Fig. 2(b) to $\mu=0.5 \mathrm{meV}$ in Fig. 1(b), and Fig. 2(c) to $\mu=4.5 \mathrm{meV}$ in Fig. 1(b). The different conductance traces in each panel correspond to increasing barrier heights $V_{0}$ at the interface; higher $V_{0}$ corresponding to the lower value of the conductance. The vertical grey bars in Fig. 2 are the topological region boundaries from Fig. 1.

It is remarkable that the interface barrier $V_{0}$ strongly quenches the conductance of the trivial regions in Figs. 2(a) and 2(b), while it leaves almost unaffected the conductance of the topological phases. This behavior can be explained by the strong sensitivity of the Andreev reflection to the barrier in the trivial phases. On the other hand, the presence of a Majorana mode in the topological phases leads to more robust quantized conductance plateaus. The topological robustness against an interface barrier provides an interesting experimental way to discern the presence of a Majorana mode. This appealing scenario, however, is valid only for relatively low chemical potentials. As shown in Fig. 2(c), with larger chemical potentials the barrier sensitivity is no longer resolving well the trivial and topological phases; rather, it yields a different behavior at low and high fields, irrespective of the topological phase in each case. At low fields the conductance is always quenched while at high fields it always remains quantized. Besides, with larger $\mu$ there are propagating modes induced by the magnetic field beyond a certain value, indicated by the dashed line in Fig. 2(c). This opening of transmission channels 



FIG. 2. (d) (upper) Sketch of a junction with a potential barrier at the interface. (a)-(c) (lower) Junction conductance as a function of the field for $L_{y}=150 \mathrm{~nm}$. Panel (a) is for $\alpha=60 \mathrm{meV} \mathrm{nm}$ and $\mu=1.5 \mathrm{meV}$; panel (b) is for $\alpha=45 \mathrm{meV} \mathrm{nm}$ and $\mu=0.5 \mathrm{meV}$, and panel (c) is for $\alpha=45 \mathrm{meV} \mathrm{nm}$ and $\mu=4.5 \mathrm{meV}$. The different curves are for increasing barrier heights at the interface, with the corresponding value of $V_{0}$ in meV indicated near each curve. Solid vertical bars show the phase transition boundaries from Fig. 1, while the dashed bar in panel (c) signals the activation threshold for propagating modes (blue curves in Fig. 1). Majorana phase regions are indicated with an $M$. Parameters: $L_{x}=7 \mathrm{~nm}, g=15, m=0.033 m_{e}$.

causes a counterintuitive effect; a conductance decrease due to the decrease of Andreev reflection $R_{A}$.

\section{Dependence on $\boldsymbol{L}_{y}$}

We next consider the influence of varying $L_{y}$ on the above results. In wider junctions the effective energy scale set by the transverse confinement is reduced and, therefore, a fixed $\mu$ and $\alpha$ values will evolve from weak to strong regime by simply increasing $L_{y}$. The phase diagram for $L_{y}=225 \mathrm{~nm}$ is shown in Fig. 3. We notice that, within the explored window, the tendency with increasing $L_{y}$ is to shrink a sequence of topological regions towards lower fields, from two regions with $B<1.5$ T in Fig. 1(c) to three regions in Fig. 3(a). The gapless part, shown by the blue line, is enlarged with respect
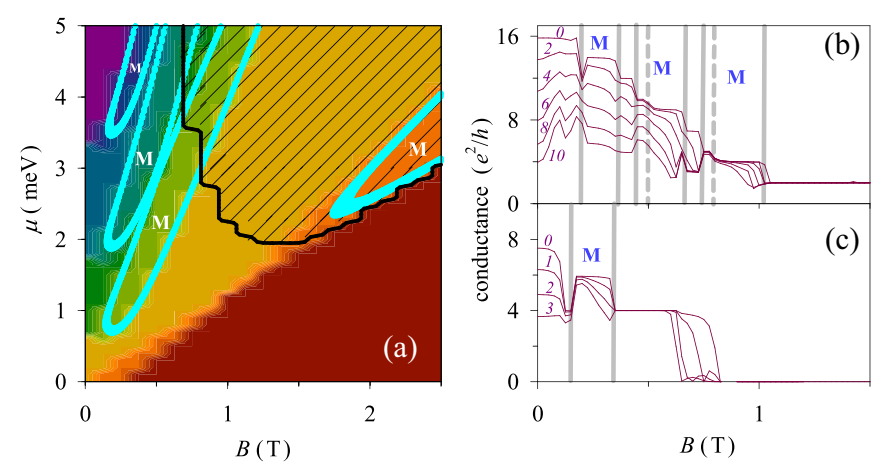

FIG. 3. (a) Topological phase diagram similar to Fig. 1(c), but for a wider junction of $L_{y}=225 \mathrm{~nm}$. The right panels show the junction conductance for (b) $\mu=4 \mathrm{meV}$ and (c) $\mu=1 \mathrm{meV}$. As in Fig. 2 the results for increasing potential barriers are displayed from upper to lower curves and the value of $V_{0}$ in $\mathrm{meV}$ is given. The Majorana phases and the activation thresholds for propagating modes are also indicated as in Fig. 2. Parameters: $\alpha=30 \mathrm{meV} \mathrm{nm}, L_{x}=7 \mathrm{~nm}$.

to Fig 1, thus leaving the protected Majorana phases only for rather small fields. Therefore, there is a severe compromise when increasing $L_{y}$ between the sequence of low field topological transitions and the gap closing for propagating modes.

Figures 3(b) and 3(c) show the conductance traces for two selected values of the chemical potential of Fig. 3(a). In these cases, the effect of the interface barrier is not clearly identifying the Majorana phases. This is similar to the result in Fig. 2(c), indicating that with larger $L_{y}$ 's the identification of robust Majorana plateaus as in Figs. 2(a) and 2(b) are not possible by simply increasing $V_{0}$. This can be understood noting that the larger $L_{y}$ implies a lower confinement energy and, thus, the requirement of low values of chemical potential already obtained in Fig. 2 becomes even more restrictive for Fig. 3. We want to stress, however, that even in cases when the interface barrier is not yielding perfectly quantized plateaus in the topological regions, the conductance still manifests a nonmonotonous $B$ dependence, usually with minima before the onset of a topological region, in Figs. 3(b) and 3(c). This anomalous magnetoconductance at low fields, as compared to a smooth decrease due to the magnetic depopulation of propagating bands, still originates in the topological transitions of the hybrid system.

With a smaller width, $L_{y}=112 \mathrm{~nm}$ in Fig. 4, the magnetoconductance scenario of topological quantized plateaus and quenched trivial regions is reinforced. In this case, the hybrid part is always gapped and the Majorana regions are more separated, for the inspected window of parameters in Fig. 4(a). Even the relatively larger chemical potential $\mu=$ $3 \mathrm{meV}$ shows a quenched conductance around $B \approx 2 \mathrm{~T}$ in Fig. 4(b) followed by a sharp increase when entering the topological phase around $B \approx 2.6 \mathrm{~T}$.

\section{Finite strip}

Although in this work we mainly focus on the NS junction, it is interesting to explore the case of a finite topological strip attached to normal contacs. That is, the case of the NSN double junction sketched in Fig. 5(a). In nontrivial phases of the finite strip, the interference between the Majorana modes 

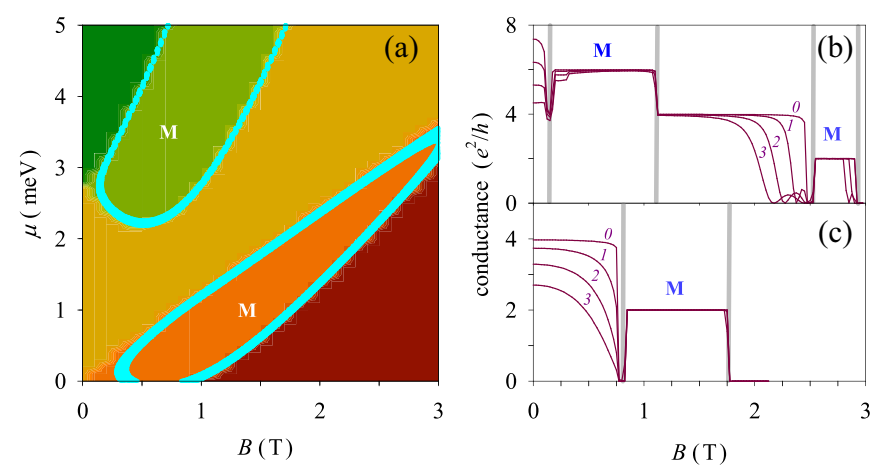

FIG. 4. Same as Fig. 3 for a narrower junction of width $L_{y}=$ $112 \mathrm{~nm}$. Panels (b) and (c) show the magnetoconductance curves for $\mu=3$ and $1 \mathrm{meV}$, respectively. Parameters: $\alpha=60 \mathrm{meV} \mathrm{nm}, L_{x}=$ $7 \mathrm{~nm}$.

on opposite ends yields energy splittings that oscillate with field and/or chemical potential. In Ref. [50] these splittings were interpreted as evidences of incipient Majorana modes in $1 \mathrm{D}$ wires.

A generalization of Eq. (5) yields the linear conductance of the NSN finite strip as [51]

$$
G=\frac{e^{2}}{h}\left(T+R_{A}\right),
$$

where $T$ is the transmission from the left to the right normal contact. Figure 5 shows the results corresponding to the same conditions of Fig. 2(a), but for finite strip lengths $L_{x}^{\prime}$
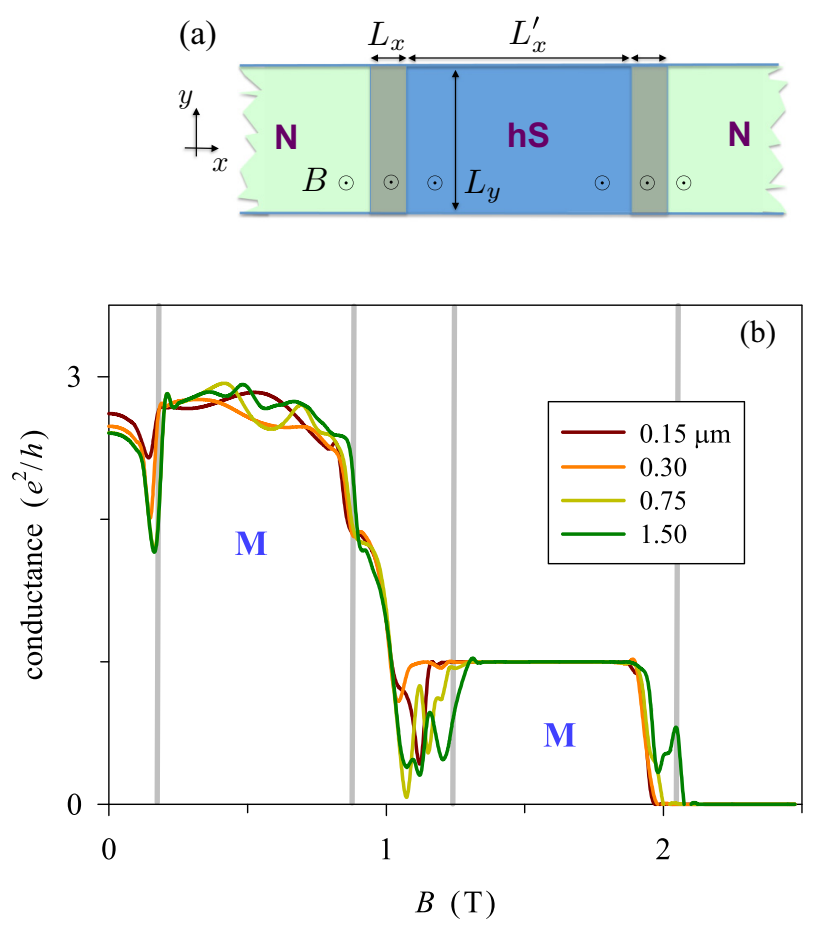

FIG. 5. (a) Sketch of the double junction formed when a finite topological strip is attached to normal contacts. (b) Strip conductance as a function of the field for the same parameters of Fig. 2(a) with a potential barrier of $2 \mathrm{meV}$. The different curves correspond to the indicated strip lengths $L_{x}^{\prime}$. from $0.15 \mu \mathrm{m}$ to $1.5 \mu \mathrm{m}$. As expected, we find a tendency towards oscillating conductance as a function of the field, with larger periods the shorter the wire. Nevertheless, the Majorana regions are still clearly visible and characterized by plateaus of enhanced conductance preceded by conductance dips. In shorter wires these dips are greatly reduced due to the increased transmission $T$. We also notice that an overall reduction of the conductance by $\approx 2$ is observed in the finite strip (Fig. 5), as compared to the single junction [Fig. 2(a)] due to the series combination of the two identical interfaces.

\section{CONCLUSION}

We calculated the phase diagrams of hybrid semiconductor-superconductor 2DEG strips in the vertical magnetic field. The $\mu-B$ plane contains Majorana regions that depend on the values of SO coupling $(\alpha)$ and transverse width $\left(L_{y}\right)$. For increasing $L_{y}$ 's or increasing $\alpha$ 's the topological regions are squeezed towards lower fields. We then investigated the magnetoconductance of the junction between a normal and a hybrid strip, in the presence of a potential barrier at the interface. For low values of the chemical potential we find a magnetoconductance scenario of robust quantized topological plateaus and quenched trivial regions, with respect to an interface potential barrier. More in general, for higher chemical potentials or transverse widths, we find that the sequence of topological regions with increasing field causes a nonmonotonous magnetoconductance, with anomalous steps and minima usually before the onset of topological regions, in the presence of an interface barrier.

Our results suggest a direct validation of the topological phases in 2D junctions by means of magnetoconductance measurements. The strong orbital magnetic effect in the perpendicular field can be used advantageously to engineer topological transitions at relatively low fields in hybrid semiconductor-superconductor 2DEG strips. We calculated a lowest critical field of $B_{c} \approx 0.2 \mathrm{~T}$ with the parameters of Fig. 3, but even lower $B_{c}$ 's would be achieved with smaller pairings $\Delta_{0}<0.3 \mathrm{meV}$ and wider junctions $L_{y}>225 \mathrm{~nm}$.

\section{ACKNOWLEDGMENTS}

We acknowledge support from MINECO (Spain) Grant No. MAT2017-82639, MINECO/AEI/FEDER María de Maeztu Program for Units of Excellence MDM2017-0711, and from EPSRC (UK) for MQIC Grant No. EP/S019324/1.

\section{APPENDIX: PHASE DIAGRAMS WITHOUT ORBITAL EFFECTS}

Since the magneto-orbital terms are often neglected in the literature, it is worth confronting our above results for the full Hamiltonian, Eq. (1), with the case of total absence of orbital contributions; that is, neglecting the last three terms depending on the magnetic length $l_{z}$ in Eq. (1). Another frequent simplifying assumption in the literature is neglecting the transverse Rashba SO term $\propto \alpha p_{y} \sigma_{x}$ in Eq. (1). This transverse SO term is the source in multiband wires for the coupling between Majorana modes of different bands, leading 




FIG. 6. Phase diagrams similar to Fig. 1 but neglecting the magneto-orbital terms in the Hamiltonian, i.e., neglecting the last three contributions of Eq. (1). Panel (a) is for the full SO interaction $\left(p_{x} \sigma_{y}-p_{y} \sigma_{x}\right)$ while panel (b) is for the case of a partial SO containing only the longitudinal $p_{x} \sigma_{y}$ contribution. Parameters: $L_{y}=150 \mathrm{~nm}, \alpha=30 \mathrm{meVnm}$. to an effective repulsion between different Majorana regions of the phase diagram.

Figure 6 confirms that in the absence of orbital effects the Majorana phases, highlighted by the cyan lines, appear at larger fields. Comparing Fig. 6(a) to the corresponding Fig. 1(c) with the same $\alpha$ and $L_{y}$, we notice the high relevance of the orbital effect on the phase diagram. While the absolute lowest critical field in Fig. 1(c) is $B_{c} \approx 0.2 \mathrm{~T}$ achieved for $\mu \approx 2$ and $4.5 \mathrm{meV}$, in Fig. 6(a) it is $B_{c} \approx 1 \mathrm{~T}$ for $\mu \approx$ $0.2 \mathrm{meV}$. Thus, orbital field effects cannot be neglected in a quantitative study of the critical fields of hybrid strips. It is also worth stressing that the whole window of parameters represented in Fig. 6(a) corresponds to a gapped spectrum (excluding, of course, the $k=0$ gap-closing lines of the phase transition boundaries); while in Fig. 1(c) there is a sizable gapless region highlighted by a thin-line hatching. The orbital terms thus favour the gap closing of the spectrum in larger regions, lowering the parameter ranges where Majorana protection is ensured. We also checked that similar modifications are obtained neglecting orbital terms in the other two panels of Fig. 1 for the larger values of $\alpha$.

Neglecting the Rashba transverse term $\alpha p_{y} \sigma_{x}$, in addition to the orbital terms, leads to the phase diagram of Fig. 6(b). In this case, the Majorana region boundaries are analytical $[42,43]$

$$
\Delta_{B}=\sqrt{\Delta_{0}^{2}+\left(\mu-\frac{\hbar^{2} \pi^{2} n^{2}}{2 m L_{y}^{2}}\right)^{2}}, n=1,2, \ldots,
$$

the condition for the absolute lowest critical field being $\Delta_{B}=$ $\Delta_{0}$ for values of the chemical potential equal to the successive transverse eigenenergies $\mu=\hbar^{2} \pi^{2} n^{2} /\left(2 m L_{y}^{2}\right)$ with $n=$ $1,2, \ldots$. Since we assumed $\Delta_{0}=0.3 \mathrm{meV}$, this corresponds in Fig. 6 (b) to $B_{c} \approx 0.7 \mathrm{~T}$ for $\mu \approx(0.5,2,4.5, \ldots) \mathrm{meV}$.
[1] V. Mourik, K. Zuo, S. M. Frolov, S. R. Plissard, E. P. A. M. Bakkers, and L. P. Kouwenhoven, Signatures of Majorana fermions in hybrid superconductor-semiconductor nanowire devices, Science 336, 1003 (2012).

[2] A. Das, Y. Ronen, Y. Most, Y. Oreg, M. Heiblum, and H. Shtrikman, Zero-bias peaks and splitting in an Al-InAs nanowire topological superconductor as a signature of Majorana fermions, Nat. Phys. 8, 887 (2012).

[3] M. T. Deng, C. L. Yu, G. Y. Huang, M. Larsson, P. Caroff, and H. Q. Xu, Anomalous zero-bias conductance peak in a Nb-InSb nanowire-Nb hybrid device, Nano Lett. 12, 6414 (2012).

[4] A. D. K. Finck, D. J. Van Harlingen, P. K. Mohseni, K. Jung, and $\mathrm{X}$. Li, Anomalous Modulation of a Zero-Bias Peak in a Hybrid Nanowire-Superconductor Device, Phys. Rev. Lett. 110, 126406 (2013).

[5] H. O. H. Churchill, V. Fatemi, K. Grove-Rasmussen, M. T. Deng, P. Caroff, H. Q. Xu, and C. M. Marcus, Superconductornanowire devices from tunneling to the multichannel regime: Zero-bias oscillations and magnetoconductance crossover, Phys. Rev. B 87, 241401(R) (2013).

[6] J. Alicea, New directions in the pursuit of Majorana fermions in solid state systems, Rep. Prog. Phys. 75, 076501 (2012).
[7] C. W. J. Beenakker, Search for Majorana fermions in superconductors, Annu. Rev. Condens. Matter Phys. 4, 113 (2013).

[8] T. D. Stanescu and S. Tewari, Majorana fermions in semiconductor nanowires: Fundamentals, modeling, and experiment, J. Phys.: Condens. Matter 25, 233201 (2013).

[9] R. Aguado, Majorana quasiparticles in condensed matter, Riv. Nuovo Cimento 40, 523 (2017).

[10] R. M. Lutchyn, E. P. A. M. Bakkers, L. P. Kouwenhoven, P. Krogstrup, C. M. Marcus, and Y. Oreg, Majorana zero modes in superconductor-semiconductor heterostructures, Nat. Rev. Mater. 3, 52 (2018).

[11] J. Osca and L. Serra, Complex band-structure analysis and topological physics of Majorana nanowires, Eur. Phys. J. B 92, 101 (2019).

[12] S. M. Albrecht, A. P. Higginbotham, M. Madsen, F. Kuemmeth, T. S. Jespersen, J. Nygård, P. Krogstrup, and C. M. Marcus, Exponential protection of zero modes in Majorana islands, Nature (London) 531, 206 (2016).

[13] M. T. Deng, S. Vaitiekenas, E. B. Hansen, J. Danon, M. Leijnse, K. Flensberg, J. Nygård, P. Krogstrup, and C. M. Marcus, Majorana bound state in a coupled quantum-dot hybrid-nanowire system, Science 354, 1557 (2016). 
[14] F. Nichele, A. C. C. Drachmann, A. M. Whiticar, E. C. T. O'Farrell, H. J. Suominen, A. Fornieri, T. Wang, G. C. Gardner, C. Thomas, A. T. Hatke, P. Krogstrup, M. J. Manfra, K. Flensberg, and C. M. Marcus, Scaling of Majorana Zero-Bias Conductance Peaks, Phys. Rev. Lett. 119, 136803 (2017).

[15] Ö. Gül, H. Zhang, J. D. S. Bommer, M. W. A. de Moor, D. Car, S. R. Plissard, E. P. A. M. Bakkers, A. Geresdi, K. Watanabe, T. Taniguchi, and L. P. Kouwenhoven, Ballistic Majorana nanowire devices, Nat. Nanotechnol. 13, 192 (2018).

[16] H. Zhang, C.-X. Liu, S. Gazibegovic, D. Xu, J. A. Logan, G. Wang, N. van Loo, J. D. S. Bommer, M. W. A. de Moor, D. Car, R. L. M. Op het Veld, P. J. van Veldhoven, S. Koelling, M. A. Verheijen, M. Pendharkar, D. J. Pennachio, B. Shojaei, J. S. Lee, C. J. Palmstrøm, E. P. A. M. Bakkers, S. Das Sarma, and L. P. Kouwenhoven, Quantized Majorana conductance, Nature (London) 556, 74 (2018).

[17] M. W. A. de Moor, J. D. S. Bommer, D. Xu, G. W. Winkler, A. E. Antipov, A. Bargerbos, G. Wang, N. van Loo, R. L. M. O. het Veld, S. Gazibegovic, D. Car, J. A Logan, M. Pendharkar, J. S. Lee, E. P. A. M. Bakkers, C. J. Palmstrøm, R. M. Lutchyn, L. P. Kouwenhoven, and H. Zhang, Electric field tunable superconductor-semiconductor coupling in Majorana nanowires, New J. Phys. 20, 103049 (2018).

[18] A. Grivnin, E. Bor, M. Heiblum, Y. Oreg, and H. Shtrikman, Concomitant opening of a bulk-gap with an emerging possible Majorana zero mode, Nat. Commun. 10, 1940 (2019).

[19] J. D. S. Bommer, H. Zhang, Ö. Gül, B. Nijholt, M. Wimmer, F. N. Rybakov, J. Garaud, D. Rodic, E. Babaev, M. Troyer, D. Car, S. R. Plissard, E. P. A. M. Bakkers, K. Watanabe, T. Taniguchi, and L. P. Kouwenhoven, Spin-Orbit Protection of Induced Superconductivity in Majorana Nanowires, Phys. Rev. Lett. 122, 187702 (2019).

[20] A. C. Potter and P. A. Lee, Majorana end states in multiband microstructures with Rashba spin-orbit coupling, Phys. Rev. B 83, 094525 (2011).

[21] R. M. Lutchyn, T. D. Stanescu, and S. Das Sarma, Search for Majorana Fermions in Multiband Semiconducting Nanowires, Phys. Rev. Lett. 106, 127001 (2011).

[22] J. S. Lim, L. Serra, R. López, and R. Aguado, Magneticfield instability of Majorana modes in multiband semiconductor wires, Phys. Rev. B 86, 121103(R) (2012).

[23] D. Rainis, L. Trifunovic, J. Klinovaja, and D. Loss, Towards a realistic transport modeling in a superconducting nanowire with Majorana fermions, Phys. Rev. B 87, 024515 (2013).

[24] P. San-Jose, E. Prada, and R. Aguado, Mapping the Topological Phase Diagram of Multiband Semiconductors with Supercurrents, Phys. Rev. Lett. 112, 137001 (2014).

[25] N. Sedlmayr, J. M. Aguiar-Hualde, and C. Bena, Majorana bound states in open quasi-one-dimensional and twodimensional systems with transverse rashba coupling, Phys. Rev. B 93, 155425 (2016).

[26] C. W. Groth, M. Wimmer, A. R. Akhmerov, and X. Waintal, Kwant: A software package for quantum transport, New J. Phys. 16, 063065 (2014).

[27] B. Nijholt and A. R. Akhmerov, Orbital effect of magnetic field on the Majorana phase diagram, Phys. Rev. B 93, 235434 (2016).

[28] A. Manolescu, A. Sitek, J. Osca, L. Serra, V. Gudmundsson, and T. Dan Stanescu, Majorana states in prismatic core-shell nanowires, Phys. Rev. B 96, 125435 (2017).
[29] B. D. Woods, J. Chen, S. M. Frolov, and T. D. Stanescu, Zeroenergy pinning of topologically trivial bound states in multiband semiconductor-superconductor nanowires, Phys. Rev. B 100, 125407 (2019).

[30] J. Shabani, M. Kjaergaard, H. J. Suominen, Y. Kim, F. Nichele, K. Pakrouski, T. Stankevic, R. M. Lutchyn, P. Krogstrup, R. Feidenhans'l, S. Kraemer, C. Nayak, M. Troyer, C. M. Marcus, and C. J. Palmstrøm, Two-dimensional epitaxial superconductor-semiconductor heterostructures: A platform for topological superconducting networks, Phys. Rev. B 93, 155402 (2016).

[31] M. Kjaergaard, F. Nichele, H. J. Suominen, M. P. Nowak, M. Wimmer, A. R. Akhmerov, J. A. Folk, K. Flensberg, J. Shabani, C. J. Palmstrøm, and C. M. Marcus, Quantized conductance doubling and hard gap in a two-dimensional semiconductor-superconductor heterostructure, Nat. Commun. 7, 12841 (2016).

[32] K. Delfanazari, R. K. Puddy, P. Ma, T. Yi, M. Cao, Y. Gul, I. Farrer, D. A. Ritchie, H. J. Joyce, M. J. Kelly, and C. G. Smith, On-chip Andreev devices: Hard superconducting gap and quantum transport in ballistic $\mathrm{Nb}-\mathrm{In}_{0.75} \mathrm{Ga}_{0.25}$ As-quantumwell-Nb Josephson junctions, Adv. Mater. 29, 1701836 (2017).

[33] K. Delfanazari, R. K. Puddy, P. Ma, T. Yi, M. Cao, C. Richardson, I. Farrer, D. A. Ritchie, H. J. Joyce, M. J. Kelly, and C. G. Smith, On-chip hybrid superconducting-semiconducting quantum circuit, IEEE Trans. Appl. Supercond. 28, 1 (2018).

[34] K. Delfanazari, P. Ma, R. Puddy, T. Yi, M. Cao, Y. Gul, C. L. Richardson, I. Farrer, D. Ritchie, H. J. Joyce, M. J. Kelly, and C. G. Smith, Scalable quantum integrated circuits on superconducting two-dimensional electron gas platform, J. Vis. Exp. 150, e57818 (2019).

[35] A. Fornieri, A. M. Whiticar, F. Setiawan, E. Portolés, A. C. C. Drachmann, A. Keselman, S. Gronin, C. Thomas, T. Wang, R. Kallaher, G. C. Gardner, E. Berg, M. J. Manfra, A. Stern, C. M. Marcus, and F. Nichele, Evidence of topological superconductivity in planar Josephson junctions, Nature 569, 89 (2019).

[36] H. Ren, F. Pientka, S. Hart, A. T. Pierce, M. Kosowsky, L. Lunczer, R. Schlereth, B. Scharf, E. M. Hankiewicz, L. W. Molenkamp, B. I. Halperin, and A. Yacoby, Topological superconductivity in a phase-controlled Josephson junction, Nature (London) 569, 93 (2019).

[37] J. S. Lim, R. Lopez, and L. Serra, Emergence of Majorana modes in cylindrical nanowires, Europhys. Lett. 103, 37004 (2013).

[38] O. Dmytruk and J. Klinovaja, Suppression of the overlap between Majorana fermions by orbital magnetic effects in semiconducting-superconducting nanowires, Phys. Rev. B 97, 155409 (2018).

[39] J. Osca and L. Serra, Majorana states and magnetic orbital motion in planar hybrid nanowires, Phys. Rev. B 91, 235417 (2015).

[40] M. P. Nowak and P. Wójcik, Renormalization of the Majorana bound state decay length in a perpendicular magnetic field, Phys. Rev. B 97, 045419 (2018).

[41] P. Wójcik and M. P. Nowak, Durability of the superconducting gap in Majorana nanowires under orbital effects of a magnetic field, Phys. Rev. B 97, 235445 (2018).

[42] R. M. Lutchyn, J. D. Sau, and S. Das Sarma, Majorana Fermions and a Topological Phase Transition in 
Semiconductor-Superconductor Heterostructures, Phys. Rev. Lett. 105, 077001 (2010).

[43] Y. Oreg, G. Refael, and F. von Oppen, Helical Liquids and Majorana Bound States in Quantum Wires, Phys. Rev. Lett. 105, 177002 (2010).

[44] J. Klinovaja and D. Loss, Composite Majorana fermion wave functions in nanowires, Phys. Rev. B 86, 085408 (2012).

[45] R. B. Lehoucq, D. C. Sorensen, and C. Yang, ARPACK Users Guide: Solution of Large-Scale Eigenvalue Problems with Implicitly Restarted Arnoldi Methods (SIAM, Philadelphia, 1998).

[46] J. Osca and L. Serra, Current distributions in stripe Majorana junctions, Eur. Phys. J. B 90, 28 (2017).

[47] G. E. Blonder, M. Tinkham, and T. M. Klapwijk, Transition from metallic to tunneling regimes in superconducting microconstrictions: Excess current, charge imbalance, and supercurrent conversion, Phys. Rev. B 25, 4515 (1982).

[48] K. Takase, Y. Ashikawa, G. Zhang, K. Tateno, and S. Sasaki, Highly gate-tuneable Rashba spin-orbit interaction in a gate-allaround InAs nanowire metal-oxide-semiconductor field-effect transistor, Sci. Rep. 7, 930 (2017).

[49] M. Diez, J. P. Dahlhaus, M. Wimmer, and C. W. J. Beenakker, Andreev reflection from a topological superconductor with chiral symmetry, Phys. Rev. B 86, 094501 (2012).

[50] S. Das Sarma, J. D. Sau, and T. D. Stanescu, Splitting of the zero-bias conductance peak as smoking gun evidence for the existence of the majorana mode in a superconductorsemiconductor nanowire, Phys. Rev. B 86, 220506(R) (2012).

[51] J. S. Lim, R. López, and L. Serra, Transport through Majorana nanowires attached to normal leads, New J. Phys. 14, 083020 (2012). 